\title{
TWO-PROTON CORRELATIONS FROM PB+PB CENTRAL COLLISIONS
}

\author{
F. Wang (for the NA49 Collaboration) \\ Nuclear Science Division, Lawrence Berkeley National Laboratory \\ One Cyclotron Road, Berkeley, CA 94720, USA \\ FQWang@lbl.gov
}

\begin{abstract}
The two-proton correlation function at midrapidity from $\mathrm{Pb}+\mathrm{Pb}$ central collisions at $158 \mathrm{AGeV}$ has been measured by the NA49 experiment. The preliminary results are compared to model predictions from proton source distributions of static thermal Gaussian sources and the transport models of RQMD and VENUS. We obtain an effective proton source size $\sigma_{\text {eff }}=4.0 \pm 0.15$ (stat.) ${ }_{-0.18}^{+0.06}$ (syst.) fm. The RQMD model underpredicts the correlation function $\left(\sigma_{\text {eff }}=4.41 \mathrm{fm}\right)$, while the vENus model overpredicts the correlation function $\left(\sigma_{\text {eff }}=3.55 \mathrm{fm}\right)$.
\end{abstract}

Keywords: two-proton correlation, heavy ion

Nuclear matter under extreme conditions of high energy density has been extensively studied through high energy heavy ion collisions. The baryon density plays an important role in the dynamical evolution of these collisions. To measure the baryon spatial density, one needs information on the space-time extent of the baryon source. The spacetime extent of the proton source at freeze-out can be inferred from twoproton correlation functions. The peak in the correlation function at $q_{\text {inv }}=\sqrt{-q_{\mu} q^{\mu}} / 2 \approx 20 \mathrm{MeV} / c$ is inversely related to the effective size of the proton source $[1,2]$. In the above, $q_{\mu}$ is the difference of the proton 4-momenta, and $q_{\text {inv }}$ is the momentum magnitude of one proton in the rest frame of the pair.

We report the first, preliminary results on the two-proton correlation function in the midrapidity region from $\mathrm{Pb}+\mathrm{Pb}$ central collisions at 158 $\mathrm{GeV}$ per nucleon. The measurement was done by the NA49 experi- 
ment [3] at the SPS, using the $5 \%$ most central events, corresponding to collisions with impact parameter $b \leq 3.3 \mathrm{fm}$.

Two independent analyses were performed on the data: $d E / d x$ analysis which used particles in the rapidity range $2.9<y<3.4$ (assuming proton mass) with at least $70 \%$ probability to be a proton obtained from their ionization energies deposited in the time projection chambers (TPCs), and TOF analysis which used identified protons by combining the time of flight information and the $d E / d x$. Both analyses used protons up to a transverse momentum $p_{T}=2 \mathrm{GeV} / c$.

The proton samples are contaminated by weak decay protons $\left(\Lambda+\Sigma^{0}\right.$ and $\left.\Sigma^{+}\right)$which are incorrectly reconstructed as primary vertex tracks. From the measured single particle distributions [4, 5] and model calculations of RQMD and VENUS, we estimate the contamination to be $15_{-5}^{+15} \%$. This results in about $30_{-10}^{+20} \%$ of the proton pairs having at least one proton from weak decays. These protons do not have correlation with protons from the primary interactions. In the $d E / d x$ analysis, further contamination is present from kaons on the lower tail of their $d E / d x$ distribution merging into the region where particles have at least $70 \%$ probability to a proton. This resulted in $25 \% K^{+} p$ pairs and fewer than $2 \% K^{+} K^{+}$pairs in the proton pair sample. The coulomb hole of $K^{+} p$ pairs does not affect the resulting two-proton correlation function in the interested low $q_{\text {inv }}$ region below $50 \mathrm{MeV} / c$.

The two-proton correlation function is obtained as the ratio of the $q_{\text {inv }}$ distribution of true proton pairs to that of mixed-event pairs with protons from different events. The number of mixed-event pairs was large enough so that the statistical error on the correlation function is dominated by the statistical uncertainty in the number of true pairs. To eliminate the effect of close pair reconstruction inefficiency, a cut of $2 \mathrm{~cm}$ was applied on the pair distance at the middle plane of the TPC for both true and mixed-event pairs.

The correlation functions obtained from the two analyses can be directly compared because of the nearly symmetric acceptances about midrapidity $y_{\text {c.m. }}=2.9$. The correlation functions (with the $K^{+} p$ contamination corrected in the $d E / d x$ analysis) are consistent. In the results reported below, the true pairs and the respective mixed-event pairs from the two analyses were combined. The combined sample had about $10^{5}$ pairs with $q_{\text {inv }}<120 \mathrm{MeV} / c, 75 \%$ of which were from the TOF analysis. The $q_{\text {inv }}$ distributions of the true pairs and the mixed-event pairs are shown in the top panel of Fig. 1.1. The number of mixed-event pairs was normalized to that of true pairs in the range $q_{\text {inv }}>500 \mathrm{MeV} / c$. The resulting correlation function is shown in the bottom panel of Fig. 1.1. The prominent peak at $q_{\text {inv }} \approx 20 \mathrm{MeV} / c$ is evident. There is a statisti- 
cally significant structure in the correlation function at $q_{\text {inv }} \approx 70 \mathrm{MeV} / c$. Many systematic effects have been studied; none has been identified that can account for the structure. There have been suggestions that a sharp edge in the two-proton density distribution of the source can produce such an effect [6].

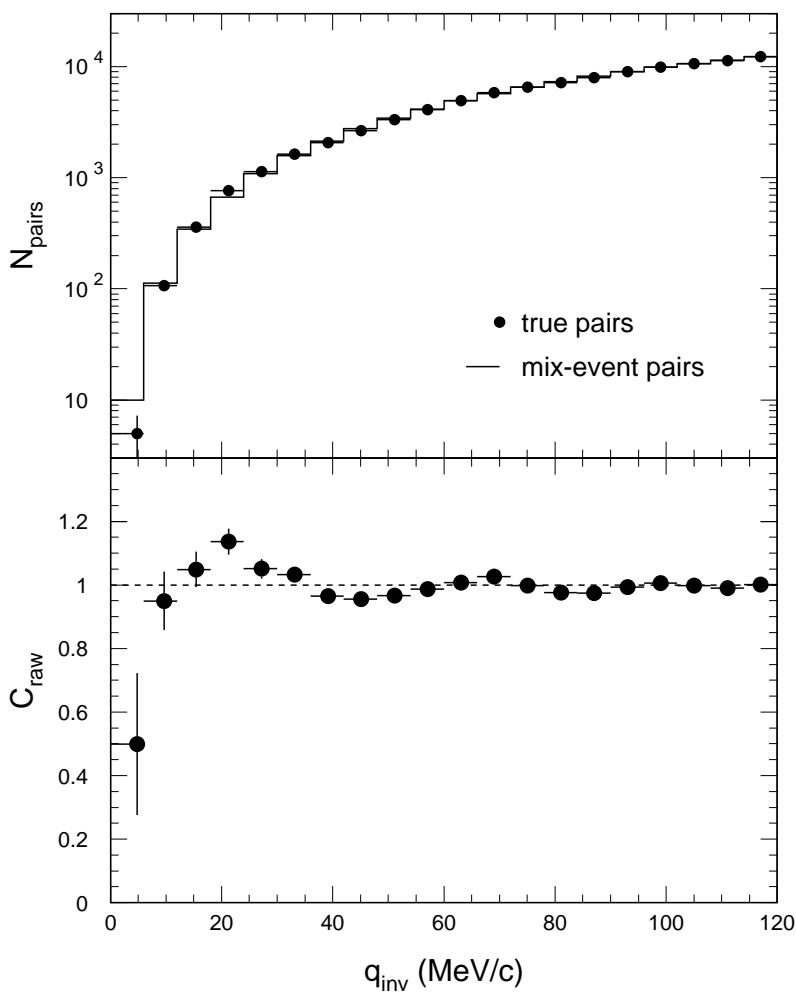

Figure 1.1 Top: $q_{\text {inv }}$ distributions of true proton pairs (points) and mixedevent proton pairs (histogram). Bottom: the measured two-proton correlation function. The contamination from weak decay protons and the finite momentum resolution were not corrected. The errors shown are statistical only.

We correct the measured correlation function for the contamination from weak decay protons and for the finite momentum resolution. The effect of the momentum resolution is only significant in the first two data points of the measured correlation function. The corrected correlation function is plotted in Fig. 1.2 as filled points.

In order to assess the proton freeze-out conditions, we compare the measured two-proton correlation function to theoretical calculations. Given the proton phase space density distribution, the two-proton correlation function can be calculated by the Koonin-Pratt Formalism [1, 7]. Two types of proton source were used:

(I) Isotropic Gaussian sources of widths $\sigma_{x, y}, \sigma_{z}$ and $\sigma_{t}$ for the space and time coordinates of protons in the source rest frame, and thermal momentum distribution of temperature $T$. No correlation between space-time and momentum of the protons is present. Following com- 
binations of parameters were used in the calculations: $\sigma_{x, y}=\sigma_{z}=\sigma$, $\sigma_{t}=0$ and $\sigma$, and $T=120 \mathrm{MeV}$ (as derived in [8]), $300 \mathrm{MeV}$ (measured inverse slope of proton transverse mass spectrum [4]) and $70 \mathrm{MeV}$ (inverse slope observed at low energy, as an extreme).

(II) Protons generated for $\mathrm{Pb}+\mathrm{Pb}$ central collisions $(b \leq 3.3 \mathrm{fm})$ at $158 \mathrm{AGeV}$ by two microscopic transport models: the Relativistic Quantum Molecular Dynamics (RQMD) model (version 2.3) [9] and the VENUS model (version 4.12) [10]. Both models describe a variety of experimental data on single particle distributions reasonably well. Protons at freeze-out have correlations between space-time and momentum intrinsic to the dynamical evolution in the models.

Only protons in the experimental acceptance are used to calculate the two-proton correlation functions, the results of which are shown in Fig. 1.2 for RQMD, VENUS, and the Gaussian source with $\sigma_{x, y}=\sigma_{z}=$ $\sigma_{t}=3.8 \mathrm{fm}$ and $T=120 \mathrm{MeV}$, respectively. While vENUS overpredicts the amplitude of the correlation function, RQMD slightly underpredicts the amplitude.

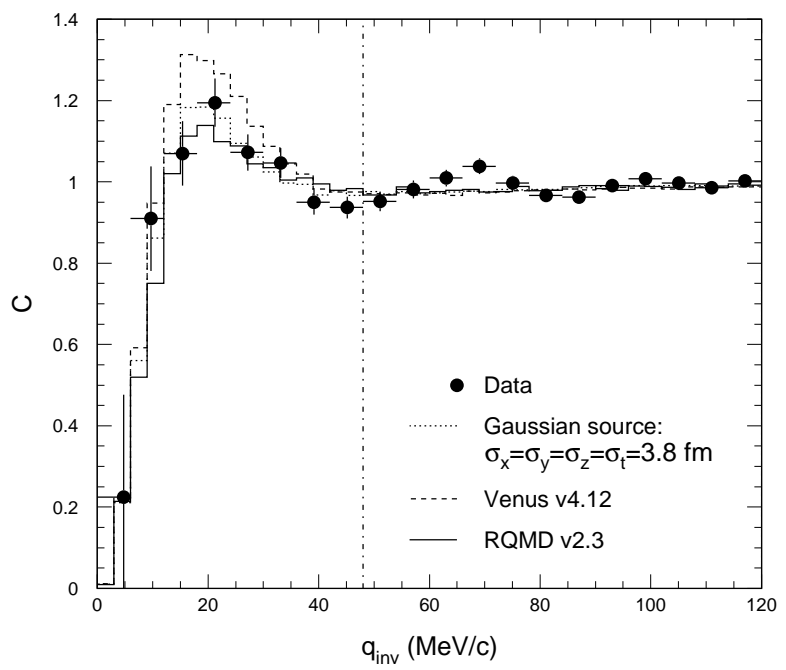

Figure 1.2 The twoproton correlation function after corrections for the $30 \%$ contamination due to weak decay protons and the finite momentum resolution (points), compared to calculations for a Gaussian source (dotted), and for freeze-out protons from RQMD v2.3 (solid) and VENUS v4.12 (dashed). The errors shown on the data points are statistical only.

We use $\chi^{2} / \mathrm{ndf}$, the normalized mean square of the point-to-point difference between the data and the calculation in the range $q_{\text {inv }}<$ $48 \mathrm{MeV} / c$ (i.e., 8 data points or ndf $=8$ ), to quantify how well the calculations agree with data. We characterize the effective size of the proton source from each model by $\sigma_{\text {eff }}=\sqrt[3]{\sigma_{\Delta x} \cdot \sigma_{\Delta y} \cdot \sigma_{\Delta z}} / \sqrt{2}$, where $\sigma_{\Delta x}, \sigma_{\Delta y}$ and $\sigma_{\Delta z}$ are the Gaussian widths fitted to the distributions in $\Delta x, \Delta y$ and $\Delta z$, distance between the protons of close pairs with $q_{\text {inv }}<48 \mathrm{MeV} / c$. The distance is evaluated in the pair rest frame at the time when the later particle freezes out [11]. Respectively for RQMD and 
VENUs, the $\chi^{2} /$ ndf values are 1.47 and 2.29; the fitted Gaussian widths are $\left(\sigma_{\Delta x}, \sigma_{\Delta y}, \sigma_{\Delta z}\right)=(5.91,6.00,6.83) \mathrm{fm}$ and $(4.57,4.57,6.08) \mathrm{fm}$, where $z$ is the longitudinal coordinate; the resulting effective sizes are $\sigma_{\text {eff }}=$ $4.41 \mathrm{fm}$ and $3.55 \mathrm{fm}$.

In Fig. 1.3 , we study the $\chi^{2} /$ ndf as a function of $\sigma_{\text {eff }}$. The $\chi^{2} /$ ndf values from all three models follow roughly the same solid line, drawn through the points for the Gaussian sources with $T=120 \mathrm{MeV}$ to guide the eye [12]. From the minimum $\chi^{2} /$ ndf point and the points where $\chi^{2} /$ ndf has increased by 0.125 (note ndf $=8$ ), we extract $\sigma_{\text {eff }}=$ $(4.0 \pm 0.15) \mathrm{fm}$, where $0.15 \mathrm{fm}$ is the statistical error [13]. By applying a correction to the measured correlation function using a proton pair contamination of $20 \%$ and $50 \%$, we obtain a systematic error of ${ }_{-0.18}^{+0.06} \mathrm{fm}$ on $\sigma_{\text {eff }}$. We note that $\sigma_{\text {eff }}=4.0 \mathrm{fm}$ corresponds to a uniform density hard sphere of radius $\sqrt{5} \sigma_{\text {eff }}=8.9 \mathrm{fm}$.

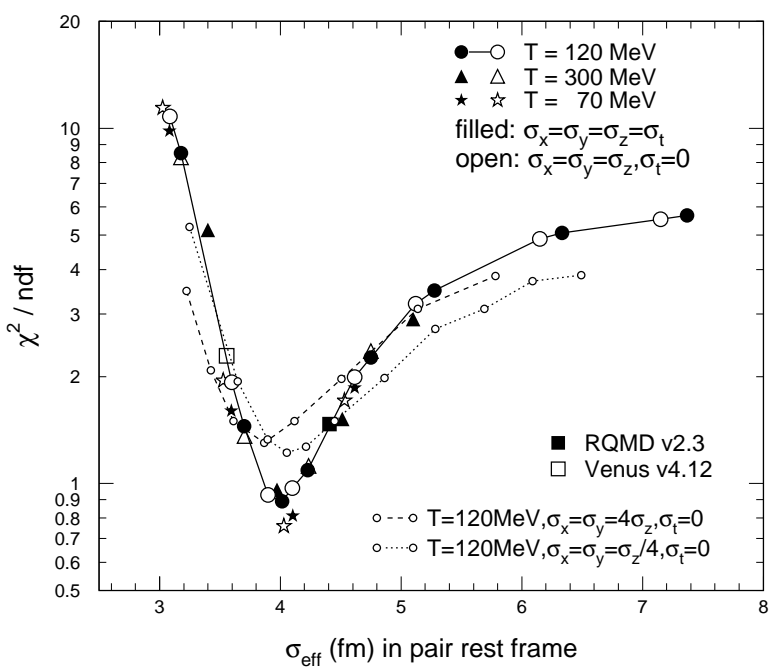

Figure 1.3 The $\chi^{2} / \mathrm{ndf}$ values as function of the effective source size $\sigma_{\text {eff }}$ for various model calculations with respect to the measured correlation function. The model calculations are for Gaussian sources (circles, triangles, and stars) and for freeze-out protons generated by RQMD v2.3 (filled square) and VENUS v4.12 (open square).

We have also studied Gaussian sources with extreme shapes: oblate $\sigma_{x, y}=4 \sigma_{z}$ and prolate $\sigma_{x, y}=\sigma_{z} / 4$ (both with $\sigma_{t}=0$ ). The corresponding $\chi^{2} /$ ndf versus $\sigma_{\text {eff }}$ curves are shown in Fig. 1.3 as dashed and dotted lines, respectively. They do not fall along the solid line, implying that the two-proton correlation function has certain sensitivity to the shape of the proton source. In principle, multi-dimensional two-proton correlation functions could reveal the shape of the proton source. However, the lack of statistics has prevented us from drawing conclusions.

We note that there is no simple relation between $\sigma_{\text {eff }}$ and the proton source in RQMD and VENUS model. From single proton distributions at freeze-out, we obtain the following Gaussian widths in the source rest frame: $\left(\sigma_{x}, \sigma_{y}, \sigma_{z}, \sigma_{t}\right)=(7.6,7.7,6.4,7.0) \mathrm{fm}$ for RQMD and 
$(3.6,3.6,4.3,1.9) \mathrm{fm}$ for venUs. The two-proton correlation function, therefore, appears to measure a smaller region of the source, i.e., $\sigma_{\Delta i}<$ $\sqrt{2} \sigma_{i}(i=x, y, z)$, due to space-time-momentum correlation. The effect is more dramatic in RQMD than in VENUS, which is consistent with the expectation that more secondary particle interactions in RQMD result in a stronger correlation between space-time and momentum of freeze-out protons. The fact that the $\chi^{2} /$ ndf values versus $\sigma_{\text {eff for RQMD and VENUS }}$ lie on the curves obtained for the isotropic Gaussian sources, in which no space-time-momentum correlation is present, suggests that the effect of the space-time-momentum correlation is small in $\sigma_{\text {eff }}$.

Finally, we comment on our two-proton correlation function in the context of other measurements. The pion source size measured by interferometry increases with the pion multiplicity [14], which increases steadily with bombarding energy in similar colliding systems [15]. Due to the large pion-nucleon cross-section, one would expect that protons and pions freeze-out under similar conditions, therefore, the proton source size would increase with bombarding energy as well. However, our measurement, in conjunction with preliminary results obtained at GSI [16] and AGS [17] energies, shows that the peak height is rather insensitive to the bombarding energy. This implies that the effective sizes of the freeze-out proton sources are similar in heavy ion collisions over a wide energy range. More detailed studies are needed to understand the possible acceptance and instrumental effects in these measurements.

In summary, the NA49 experiment has measured the two-proton correlation function at midrapidity from $\mathrm{Pb}+\mathrm{Pb}$ central collisions at 158 $\mathrm{GeV}$ per nucleon. From comparisons between the data and the calculations, we extract an effective proton source size of $\sigma_{\text {eff }}=4.0 \pm$ 0.15 (stat.) ${ }_{-0.18}^{+0.06}$ (syst.) fm. The RQMD model underpredicts the amplitude of the correlation function $\left(\sigma_{\text {eff }}=4.41 \mathrm{fm}\right)$, while the vENUs model overpredicts the amplitude $\left(\sigma_{\text {eff }}=3.55 \mathrm{fm}\right)$. Due to the space-timemomentum correlation, the two-proton correlation function is sensitive only to a limited region of the proton source. Our measurement together with the measurements at lower energies suggest a very weak dependence of the two-proton correlation function on bombarding energy.

Acknowledgment: I would like to thank M. Cristinziani who did part of the data analysis. Fruitful discussions with Drs. P.M. Jacobs, R. Lednicky, S. Panitkin, A.M. Poskanzer, H.G. Ritter, P. Seyboth, S. Voloshin and N. Xu are greatly acknowledged. This work was partially supported by the Director, Office of Energy Research, Division of Nuclear Physics of the Office of High Energy and Nuclear Physics of the US Department of Energy under Contract DE-AC03-76SF00098. 


\section{References}

[1] S.E. Koonin, Phys. Lett. 70B, 43 (1977).

[2] D.H. Boal, C. Gelbke, and B.K. Jennings, Rev. Mod. Phys. 62, 553 (1990); R. Lednicky and V.L. Lyuboshits, Sov. J. Nucl. Phys. 35, 770 (1982).

[3] S. Wenig (NA49 Coll.), NIM A409, 100 (1998); S. Afanasiev et al. (NA49 Coll.), CERN-EP/99-001 (1999), NIM in press.

[4] H. Appelshäuser et al. (NA49 Coll.), nucl-ex/9810014 (1998), Phys. Rev. Lett. in press.

[5] C. Bormann et al. (NA49 Coll.), J. Phys. G23, 1817 (1997).

[6] M.I. Podgoretsky, Sov. J. Part. Nucl. 20, 266 (1989); A. Makhlin and E. Surdutovich, hep-ph/9809278 (1998); D. Brown and E. Shuryak, private communications.

[7] S. Pratt and M.B. Tsang, Phys. Rev. C 36, 2390 (1987).

[8] H. Appelshäuser et al. (NA49 Coll.), Eur. Phys. J. C2, 661 (1998).

[9] H. Sorge, H. Stocker, and W. Greiner, Ann. Phys. 192, 266 (1989); H. Sorge et al., Phys. Lett. B 289, 6 (1992); H. Sorge, H. Stöcker, and W. Greiner, Nucl. Phys. A498, 567c (1989); H. Sorge, Phys. Rev. C 52, 3291 (1995).

[10] K. Werner, Phys. Rep. 232, 87 (1993).

[11] For a chaotic Gaussian source, $\sigma_{\text {eff }}$ is close to the non-relativistic approximation of the effective size in the source rest frame, $\sqrt[6]{\left(\sigma_{x, y}^{2}+\frac{T}{2 m} \sigma_{t}^{2}\right)^{2} \cdot\left(\sigma_{z}^{2}+\frac{T}{2 m} \sigma_{t}^{2}\right)}$, where $\frac{T}{m}$ is the one-dimensional mean squared velocity of the thermal protons, and $\frac{T}{2 m}$ is that of the close pairs.

[12] The shape of the $\chi^{2} /$ ndf versus $\sigma_{\text {eff }}$ curve can be understood as follows: the $\chi^{2} /$ ndf approaches an asymptotic value for large sources to which the two-proton correlation function is not sensitive to the source size any more, whereas for small sources, the two-proton correlation function is very sensitive and its strength decreases rapidly with increasing source size.

[13] Particle Data Group, Eur. Phys. J. C3, 172-177 (1998).

[14] K. Kaimi et al. (NA44 Coll.), Z. Phys. C 75, 619 (1997); I.G. Bearden et al. (NA44 Coll.), Phys. Rev. C 58, 1656 (1998); M.D. Baker (E802 Coll.), Nucl. Phys. A610, 213c (1996).

[15] M. Gazdzicki and D. Roehrich, Z. Phys. C 65, 215 (1995).

[16] C. Schwarz et al. (ALADIN Coll.), nucl-ex/9704001 (1997); R. Fritz et al. (ALADIN Coll.), nucl-ex/9704002 (1997). 
[17] S. Panitkin et al. (E895 Coll.), BNL/E895 preliminary. 